Chinese Journal of Organic Chemistry

NOTE

\title{
二取代烯基硼酸的氯化反应研究
}

\author{
徐 衍 ${ }^{a}$ 李战雄 ${ }^{a}$ 李 慧 $b$ 杨 军*,b \\ ( ${ }^{a}$ 苏州大学纺织与服装工程学院 苏州 215021) \\ $\left({ }^{b}\right.$ 中国科学院上海有机化学研究所 上海 200032)
}

\begin{abstract}
摘要 由二取代烯基硼酸[4-substituted-1,2-oxaborol-2(5H)-ol] 与 $N$-氯代丁二酰亚胺(NCS)在甲醇中常温反应 $0.5 \mathrm{~h}$, 可以 中等到良好的收率构型保持地合成得到取代的(Z)-3-氯烯丙醇, 产物经 ${ }^{1} \mathrm{H}$ NMR, ${ }^{13} \mathrm{C}$ NMR, FT-IR, MS 和 HRMS 鉴定. 通过该方法合成取代的 $(Z)-3$-氯烯丙醇操作简便, 反应条件温和, 收率较高且反应构型能够得到保持.

关键词 合成; 烯基硼酸; 卤代反应; $(Z)-2$-取代-3-氯烯丙醇
\end{abstract}

\section{Chlorination Reaction of Disubstituted Alkenylboronic Acids}

\author{
$\mathrm{Xu}, \mathrm{Yan}^{a}$ \\ Li, Zhanxiong ${ }^{a}$ \\ $\mathrm{Li}, \mathrm{Hui}^{b}$ \\ Yang, Jun ${ }^{*, b}$ \\ ( ${ }^{a}$ College of Textile and Clothing Engineering, Soochow University, Suzhou 215021) \\ $\left({ }^{b}\right.$ Shanghai Institute of Organic Chemistry, Chinese Academy of Sciences, Shanghai 200032)
}

\begin{abstract}
The chlorination reaction of 4-substituted-1,2-oxaborol-2(5H)-ols with $N$-chlorosuccinimide (NCS) afford (Z)-2-substituted-3-chloroallyl alcohol in moderate to good yields under the optimized condition. The structures of the products were identified by ${ }^{1} \mathrm{H}$ NMR, ${ }^{13} \mathrm{C}$ NMR, FT-IR and MS techniques. This method is simple, mild, high yield and the configuration is maintained.

Keywords synthesis; alkenylboronic acid; chlorination; (Z)-2-substituted-3-chloro allyl alcohol
\end{abstract}

(Z)-3-氯烯丙醇及其衍生物是有机合成中的一种有 价值的中间体 ${ }^{[1,2]}$, 其中 $(Z)-3$-氯-2-芳基-烯丙醇结构既 存在于天然藻类代谢产物之中 ${ }^{[3]}$, 又有专利报道其可作 为高分子材料的键合剂、单体 ${ }^{[4]}$. 文献报道中其合成方 法多通过取代的 1,2,3-三氯丙烷加热脱除 $\mathrm{HCl}$ 得到, 但 所得产物多为顺反混合物. 通过硼氢化反应可以高立体 选择性地获得烯基硼化物, 文献中报道了利用简单烯基 硼酸的卤代反应制备卤代烯烃的方法 ${ }^{[5,6]}$. 近期我们小 组 ${ }^{[7]}$ 报道了立体专一地制备二取代烯基硼酸 [4-substituted-1,2-oxaborol-2(5H)-ol] 的合成方法，所得烯基硼酸 为顺式且有两个取代基团. 在此基础上我们开展了二取 代烯基硼酸的氯化反应研究.

\section{1 实验部分}

\section{1 仪器与试剂}

Varian Mercury-400 型核磁共振仪 $\left(\mathrm{CDCl}_{3}\right.$ 为溶剂, TMS 为内标); Perkin-Elmer 983 型傅立叶红外光谱仪
(KBr 压片); HP5973N 型质谱仪; Finnigan MAT 8430 型 质谱仪.

二取代烯基硼酸根据文献[7]方法合成; $N$-氯代丁二 酰亚胺(NCS), 分析纯，上海大和化学品有限公司; 三 乙胺, 分析纯, 上海联试化工有限公司; 其余试剂均为 市售分析纯，使用前未经纯化处理. 柱层析硅胶用硅胶 300 400 目; 制备板层析用硅胶 GF 254, 烟台江友硅胶 开发有限公司.

\section{2 合成操作}

反应管中加入 $6.0 \mathrm{~mL}$ 甲醇, $0.80 \mathrm{mmol}$ 烯基硼酸和 NCS (118 mg, $0.88 \mathrm{mmol})$, 用注射器逐渐滴加三乙胺 (123 mmL, $0.88 \mathrm{mmol})$, 滴加时间不少于 $15 \mathrm{~min}$, 继续 摚拌 $30 \mathrm{~min}$. 旋去甲醇溶液, 加乙醚后硅藻土过滤, 以 无水硫酸钠干燥, 快速柱层析 $[V($ 己烷 $): V($ 乙酸乙酯 $)=$ $2: 1]$ 分离即可制得取代的 $(Z)-2$-取代-3-氯一烯丙醇.

(Z)-3-氯-2-对甲基苯基丙基-2-烯-1-醇(1a): ${ }^{1} \mathrm{H}$ NMR $\left(400 \mathrm{MHz}, \mathrm{CDCl}_{3}\right) \delta: 7.31(\mathrm{~d}, J=7.9 \mathrm{~Hz}, 2 \mathrm{H}), 7.18(\mathrm{~d}, J=$

\footnotetext{
*E-mail: yangj@mail.sioc.ac.cn

Received June 3, 2011; revised October 20, 2011; accepted November 24, 2011.

Project supported by the National Natural Science Foundation of China (Nos. 91017006, 90917017, 90817008, 20802088).

国家自然科学基金(Nos. 91017006, 90917017, 90817008, 20802088)资助项目.
} 
$7.9 \mathrm{~Hz}, 2 \mathrm{H}), 6.35$ (s, 1H), 4.71 (s, 2H), 2.37 (s, 3H); ${ }^{13} \mathrm{C}$ NMR $\left(101 \mathrm{MHz}, \mathrm{CDCl}_{3}\right) \delta: 141.78,138.23,134.59$, 129.50, 126.56, 117.41, 60.20, 21.17; IR (KBr) v: 3377, 3076, 3027, 2922, 1734, 1706, 1613, 1559, 1512, 1457, 1384, 1252, 1023, 969, 804, 786, 718, 518, $496 \mathrm{~cm}^{-1}$; MS (EI) $m / z$ (\%): 182 (49.02, $\left.\mathrm{M}^{+}\right), 115$ (100.00), 147 (72.43), 55 (57.77), 91 (44.15), 129 (43.41), 116 (29.97), 117 (28.89); HRMS calcd for $\mathrm{C}_{10} \mathrm{H}_{11} \mathrm{OCl} 182.0498$, found 182.0493.

(Z)-3-氯-2-间甲基苯基丙基-2-烯-1-醇(1b): ${ }^{1} \mathrm{H}$ NMR (300 MHz, $\left.\mathrm{CDCl}_{3}\right) \delta: 7.14 \sim 7.29(\mathrm{~m}, 4 \mathrm{H}), 6.37(\mathrm{~s}, 1 \mathrm{H})$, $4.71(\mathrm{~s}, 2 \mathrm{H}), 2.37$ (s, 3H); ${ }^{13} \mathrm{C}$ NMR (101 MHz, $\left.\mathrm{CDCl}_{3}\right) \delta$ : $142.05,138.51,137.46,129.10,128.71,127.40,123.76$, 117.97, 60.30, 21.46; IR (KBr) v: 3363, 3073, 3027, 2922, 1607, 1487, 1457, 1379, 1260, 1223, 1021, 971, 803, 774, 721, 699, 648, 594, $444 \mathrm{~cm}^{-1}$; MS (EI) $\mathrm{m} / z$ (\%): 182 (50.01, $\mathrm{M}^{+}$), 115 (100.00), 147 (78.12), 55 (49.90), 129 (45.32), 91 (43.66), 117 (32.61), 116 (25.62); HRMS calcd for $\mathrm{C}_{10} \mathrm{H}_{11} \mathrm{OCl} 182.0498$, found 182.0502 .

(Z)-3-氯-2-苯丙基-2-烯-1-醇(1c): ${ }^{1} \mathrm{H}$ NMR (300 $\left.\mathrm{MHz}, \mathrm{CDCl}_{3}\right) \delta: 7.44 \sim 7.31(\mathrm{~m}, 5 \mathrm{H}), 6.39(\mathrm{~s}, 1 \mathrm{H}), 4.73(\mathrm{~s}$, $2 \mathrm{H}) ;{ }^{13} \mathrm{C}$ NMR $\left(101 \mathrm{MHz}, \mathrm{CDCl}_{3}\right) \delta: 141.76,137.41$, 128.64, 128.14, 126.56, 118.05, 59.97; IR (KBr) v: 3357, 3077, 2949, 2889, 1602, 1495, 1465, 1465, 1443, 1386, 1251, 1078, 1030, 970, 808, 754, $696 \mathrm{~cm}^{-1}$; MS (EI) $\mathrm{m} / \mathrm{z}$ (\%): 168 (36.13, M $\left.\mathrm{M}^{+}\right), 133$ (100.00), 115 (63.76), 102 (62.88), 103 (55.79), 77 (49.26), 55 (47.35), 51 (33.72); HRMS calcd for $\mathrm{C}_{9} \mathrm{H}_{9} \mathrm{OCl} 168.0342$, found 168.0336 .

(Z)-3-氯-2-(对甲氧基苯基)丙基-2-烯-1-醇(1d): ${ }^{1} \mathrm{H}$ NMR $\left(300 \mathrm{MHz}, \mathrm{CDCl}_{3}\right) \delta: 7.39 \sim 7.31(\mathrm{~m}, 2 \mathrm{H}), 6.95 \sim$ $6.86(\mathrm{~m}, 2 \mathrm{H}), 6.32(\mathrm{~s}, 1 \mathrm{H}), 4.70(\mathrm{~s}, 2 \mathrm{H}), 3.82(\mathrm{~s}, 3 \mathrm{H}) ;{ }^{13} \mathrm{C}$ NMR $\left(101 \mathrm{MHz}, \mathrm{CDCl}_{3}\right) \delta$ : 159.73, 141.46, 129.89, 127.88, 116.74, 114.24, 60.26, 55.36; IR (KBr) v: 3396, 3075, 3002, 2956, 2935, 2837, 1608, 1569, 1512, 1464, $1289,1246,1182,969,842,814,723,621,555 \mathrm{~cm}^{-1}$; MS (EI) $\mathrm{m} / z$ (\%): 198 (100.00, $\left.\mathrm{M}^{+}\right), 132$ (63.41), 163 (58.30), 119 (54.10), 89 (53.45), 13 (51.60), 55 (43.33), 91 (41.70); HRMS calcd for $\mathrm{C}_{10} \mathrm{H}_{11} \mathrm{O}_{2} \mathrm{Cl} 198.0448$, found 198.0451.

(Z)-2-氯亚甲基-4-甲基-1-戊醇(1e): ${ }^{1} \mathrm{H}$ NMR (300 $\left.\mathrm{MHz}, \mathrm{CDCl}_{3}\right) \delta: 5.88(\mathrm{~s}, 1 \mathrm{H}), 4.29(\mathrm{~d}, J=0.5 \mathrm{~Hz}, 2 \mathrm{H})$, $2.06(\mathrm{dd}, J=7.2,0.9 \mathrm{~Hz}, 2 \mathrm{H}), 1.82(\mathrm{tt}, J=13.7,6.7 \mathrm{~Hz}$, $1 \mathrm{H}), 0.89(\mathrm{~d}, J=6.6 \mathrm{~Hz}, 6 \mathrm{H}) ;{ }^{13} \mathrm{C} \mathrm{NMR}\left(101 \mathrm{MHz}, \mathrm{CDCl}_{3}\right)$ $\delta: 140.74,114.66,59.96,42.37,26.15,22.30$; IR (KBr) $v$ : 3334, 3071, 2957, 2928, 2870, 1708, 1631, 1598, 1466, $1385,1367,1310,1228,1167,1103,1026,903,792,760$,
$646 \mathrm{~cm}^{-1}$; MS (EI) $m / z(\%): 148\left(7.58, \mathrm{M}^{+}\right), 43$ (100.00), 41 (81.31), 88 (51.92), 57 (45.14), 95 (36.60), 113 (33.72), 53 (33.18); HRMS calcd for $\mathrm{C}_{7} \mathrm{H}_{13} \mathrm{O}_{2} \mathrm{Cl} 148.0655$, found 148.0653 .

(Z)-2-氯亚甲基-1-己醇(1f): ${ }^{1} \mathrm{H}$ NMR $(300 \mathrm{MHz}$, $\left.\mathrm{CDCl}_{3}\right) \delta: 5.89(\mathrm{~s}, 1 \mathrm{H}), 4.30(\mathrm{~s}, 2 \mathrm{H}), 2.20(\mathrm{t}, J=7.5 \mathrm{~Hz}$, $2 \mathrm{H}), 1.18 \sim 1.49(\mathrm{~m}, 5 \mathrm{H}), 0.91(\mathrm{t}, J=7.2 \mathrm{~Hz}, 3 \mathrm{H}) ;{ }^{13} \mathrm{C}$ NMR (101 MHz, $\left.\mathrm{CDCl}_{3}\right) \delta: 141.73,114.06,60.15,32.80$, 29.76, 22.32, 13.85; IR (KBr) v: 3335, 3069, 2958, 2929, 2860, 1633, 1465, 1378, 1315, 1260, 1099, 1023, 980, 931, 890, 862, 800, 760; MS (EI) $m / z$ (\%): $148\left(4.81, \mathrm{M}^{+}\right), 91$ (100.00), 113 (84.59), 57 (78.27), 95 (56.37), 41 (44.45), 93 (36.90), 55 (21.96), 69 (21.87); HRMS calcd for $\mathrm{C}_{7} \mathrm{H}_{13} \mathrm{O}_{2} \mathrm{Cl} 148.0655$, found 148.0656.

\section{2 结果与讨论}

\section{1 合成条件优化}

我们首先选取 4-对甲基苯基-1,2-噁硼-2(5H)-2-醇作 为模板反应原料，通过 4-对甲基苯基-1,2-噁硼-2(5H)-2醇与 NCS 开展氯化反应条件的优化(Eq. 1), 产物(Z)-3氯-2-对甲基苯基丙基-2-烯-1-醇(1a)的收率列于表 1 .

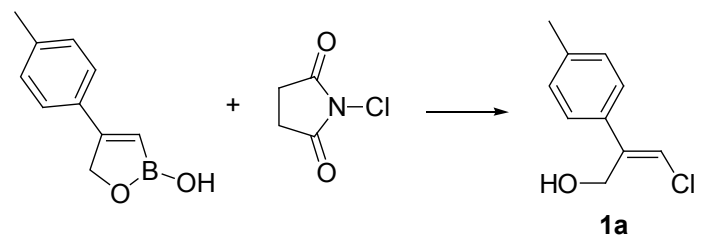

当采用氯仿作为反应的溶剂 ${ }^{[6]}$ 反应 $15 \mathrm{~min}$ 后，可通 过 TLC 跟踪发现部分原料发生反应，而延长反应时间 到 $24 \mathrm{~h}$ 后 TLC 跟踪发现仍有部分原料没有参与反应, 中止反应后测得 1a 的收率为 49\% (Entry 1). 由于氯仿 对有机硼化物溶解度较低, 我们尝试更换溶剂来提高反 应得率. 当使用极性较小的甲苯为溶剂时, $1 \mathrm{a}$ 的收率为 $45 \%$ (Entry 2); 当溶剂选择极性较大的乙腈、甲醇和 HMPA 时, 1a 的收率均有提高(Entries 3, 4, 5); 其中当溶 剂为甲醇时, $1 \mathrm{a}$ 的收率为 $85 \%$ (Entry 4); 当溶剂为水时 原料不溶, 1a 的收率仅为 33\% (Entry 6); 而当溶剂为甲 醇和水的混合溶剂 $(V: V=1: 1)$ 时, 底物的溶解度有所 改善, 1a 的收率为 $79 \%$ (Entry 7).

反应温度对此反应收率有一定影响，当反应温度从 室温降为 $0{ }^{\circ} \mathrm{C}$ 时, 1a 的收率从 85\%降至 79\% (Entry 8), 而当反应温度从室温升至回流温度时, $1 \mathrm{a}$ 的收率为 $78 \%$ (Entry 9).

在条件优化中 TLC 跟踪发现产物 1a 生成速度很快, 因此我们尝试缩短反应时间至 $15 \mathrm{~min}$, 所得 $1 \mathrm{a}$ 收率为 
表 1 二取代烯基嗍酸氯化反应条件优化 ${ }^{a}$

Table 1 The optimized condition of the chlorination reaction

\begin{tabular}{|c|c|c|c|c|c|c|}
\hline Entry & 溶剂 & 温度 & 时间 $/ \mathrm{h}$ & NCS/equiv. & 碱(equiv.) & 收率 ${ }^{b} / \%$ \\
\hline 1 & $\mathrm{CHCl}_{3}$ & 室温 & 2 & 1.1 & 三乙胺(1.1) & 49 \\
\hline 2 & Toluene & 室温 & 2 & 1.1 & 三乙胺(1.1) & 45 \\
\hline 3 & $\mathrm{CH}_{3} \mathrm{CN}$ & 室温 & 2 & 1.1 & 三乙胺(1.1) & 62 \\
\hline 4 & $\mathrm{MeOH}$ & 室温 & 2 & 1.1 & 三乙胺(1.1) & 85 \\
\hline 5 & HMPA & 室温 & 2 & 1.1 & 三乙胺(1.1) & 81 \\
\hline 6 & $\mathrm{H}_{2} \mathrm{O}$ & 室温 & 2 & 1.1 & 三乙胺(1.1) & 33 \\
\hline 7 & $\mathrm{H}_{2} \mathrm{O} / \mathrm{MeOH}(V: V=1: 1)$ & 室温 & 2 & 1.1 & 三乙胺(1.1) & 79 \\
\hline 8 & $\mathrm{MeOH}$ & $0{ }^{\circ} \mathrm{C}$ & 2 & 1.1 & 三乙胺(1.1) & 79 \\
\hline 9 & $\mathrm{MeOH}$ & 回流 & 2 & 1.1 & 三乙胺(1.1) & 78 \\
\hline 10 & $\mathrm{MeOH}$ & 室温 & 0.25 & 1.1 & 三乙胺(1.1) & 84 \\
\hline 11 & $\mathrm{MeOH}$ & 室温 & 0.5 & 1.1 & 三乙胺(1.1) & 90 \\
\hline 12 & $\mathrm{MeOH}$ & 室温 & 1 & 1.1 & 三乙胺(1.1) & 88 \\
\hline 13 & $\mathrm{MeOH}$ & 室温 & 0.5 & 2.0 & 三乙胺(1.1) & 78 \\
\hline 14 & $\mathrm{MeOH}$ & 室温 & 0.5 & 1.1 & 三乙胺(2.0) & 76 \\
\hline 15 & $\mathrm{MeOH}$ & 室温 & 0.5 & 1.1 & 二异丙胺(1.1) & 64 \\
\hline 16 & $\mathrm{MeOH}$ & 室温 & 0.5 & 1.1 & 碳酸钠(1.1) & 68 \\
\hline
\end{tabular}

${ }^{a} 0.1 \mathrm{mmol}$ 烯基嗍酸, $2 \mathrm{~mL}$ 溶剂; ${ }^{b}$ 核磁收率, 对硝基苯乙酮为内标.

$84 \%$ (Entry 10), 而当反应时间为 $0.5 \mathrm{~h}, \mathbf{1 a}$ 收率为 $90 \%$ (Entry 11), 反应时间为 $1 \mathrm{~h}, 1 \mathrm{a}$ 的收率为 $88 \%$ (Entry 12). 从上述结果可以看出当反应时间为 $0.5 \mathrm{~h}, \mathbf{1 a}$ 的收率最 高, 当进一步延长反应时间, $1 \mathrm{a}$ 的收率反而下降.

我们又探讨了 NCS 和三乙胺的用量对此反应的影 响. 当 NCS 的量增加为 2 equiv., 三乙胺的量为 1.1 equiv. 时, 1a 的收率为 78\% (Entry 13), 而当 NCS 的量为 1.1 equiv., 三乙胺的量增加到 2 equiv. 时, $\mathbf{1 a}$ 的收率为 $76 \%$ (Entry 14); 此外, 不同的碱对此反应也有影响. 当 选用无机碱碳酸钠时, $1 \mathrm{a}$ 的收率为 $64 \%$ (Entry 15), 而选 用二异丙胺作为碱时, 1a 的收率为 $68 \%$ (Entry 16).

综上, 我们确定的最优反应条件为: 在室温条件下, 在甲醇溶剂中与 1.1 equiv.的 NCS 和 1.1 equiv. 的三乙胺 反应 $0.5 \mathrm{~h}$.

\section{2 不同取代基烯基氯化物的合成}

我们随后对几种二取代的烯基硼酸在最优条件下 的氯代反应进行了底物拓展(Eq. 2), 所得各分离收率结 果见表 2 .<smiles>[R]C1=CB(O)OC1</smiles><smiles>CN1C(=O)CCC1=O</smiles><smiles>CCCCCCCC</smiles><smiles>[R]/C(=C/Cl)CO</smiles>

从表中可以看出二取代烯基硼酸均可以与 NCS 顺 利的发生氯代反应制备得到取代的 $(Z)-3$-氯一烯丙醇. 当 二取代烯基硼酸的 4 位是对甲基苯基取代基时，反应以 $83 \%$ 的分离收率得到目标产物(Entry 1); 间甲基苯基取
表 2 不同取代烯基卤化物合成 ${ }^{a}$

Table 2 The chlorination reaction of (Z)-2,2-disubstituted alkenylboronic acids

等号




\begin{tabular}{|c|c|c|c|}
\hline 序号 & 烯基硼酸 & 产物 & 收率 ${ }^{c} / \%$ \\
\hline 6 & & $1 \mathrm{f}$ & 23 \\
\hline
\end{tabular}

${ }^{a} 0.8 \mathrm{mmol}$ 烯基硼酸, $0.88 \mathrm{~mol} \mathrm{NCS}, 0.88 \mathrm{~mol}$ 三乙胺, $6 \mathrm{~mL}$ 甲醇, 室温; ${ }^{b}$ $1.2 \mathrm{mmol}$ 烯基硼酸, $1.32 \mathrm{~mol} \mathrm{NCS}, 1.32 \mathrm{~mol}$ 三乙胺, $10 \mathrm{~mL}$ 甲醇, 室温; ${ }^{c}$ 分 离收率.

代的烯基硼酸收率为 73\% (Entry 2); 苯基取代的烯基喼 酸能以 $76 \%$ 的收率得到目标产物(Entry 3); 当取代基是 对甲氧基苯时, 收率下降为 66\% (Entry 4); 当取代基分 别是异丁基和丁基时反应同样能够进行, 以 $51 \%$ 和 $23 \%$ 的收率分别得到(Z)-2-氯亚甲基-4-甲基-1-戊醇和( $Z$ )-2氯亚甲基-1-己醇(Entries 5,6). 由上可知, 烷基取代的 烯基硼酸氯化反应的收率均较芳基取代的烯基硼酸低, 而异丁基取代的烯基嗍酸氯化反应的收率较丁基取代 的烯基硼酸高，具体原因有待进一步深入的研究.

通过化合物(Z)-2-氯亚甲基-1-已醇(1f)的二维 ${ }^{1} \mathrm{H}-{ }^{1} \mathrm{H}$ NOESY 谱来确定其构型(Scheme 1, 谱图见辅助材料图 $\mathrm{S} 1)$, 化合物 $\mathbf{1 f}$ 的 ${ }^{1} \mathrm{H}$ NMR $\left(300 \mathrm{MHz}, \mathrm{CDCl}_{3}\right) \delta: 5.89$ (s, $1 \mathrm{H}), 4.30$ (s, 2H), 2.20 (t, J=7.5 Hz, 2H), $1.18 \sim 1.49(\mathrm{~m}$, $5 \mathrm{H}), 0.91(\mathrm{t}, J=7.2 \mathrm{~Hz}, 3 \mathrm{H})$; 其中化合物 $\mathbf{1 f}$ 烯氢 $\left(\mathrm{H}_{\mathrm{a}}\right)$ 化 学位移为 $\delta 5.89$, 丁基中烯丙位氢 $\left(\mathrm{H}_{\mathrm{b}}\right)$ 化学位移为 $\delta$ 2.20 , 羟甲基氢 $\left(\mathrm{H}_{\mathrm{c}}\right)$ 化学位移为 $\delta 4.30 .{ }^{1} \mathrm{H}-{ }^{1} \mathrm{H}$ NOESY 谱 分析表明 $\mathrm{H}_{\mathrm{a}}$ 与 $\mathrm{H}_{\mathrm{b}}$ 有相互作用, 因此化合物结构应为顺 式，通过与原料 4-丁基-1,2-噁硼-2(5H)-2-醇的构型相对 比可知该反应中双键的构型得到保持.<smiles>[H][R]([H])([2H])C(O)/C(O)=C/Cl</smiles>

Scheme 1

\section{3 结论}

二取代烯基硼酸与 NCS 的氯化反应可以制备取代 的(Z)-3-氯烯丙醇. 通过溶剂、反应时间、温度、物料配 比、碱对该反应的研究确定了最优反应条件为: 在室温 条件下, 在甲醇溶剂中与 1.1 equiv.的 NCS 和 1.1 equiv. 的三乙胺反应 $0.5 \mathrm{~h}$.

将上述最优反应条件应用于底物拓展后, 含有苯 基、烷基取代基的烯基硼酸与 $\mathrm{NCS}$ 发生氯化反应后都 能以中等到良好的收率得到相应取代的(Z)-3-氯烯丙醇, $\mathrm{IR},{ }^{1} \mathrm{H} \mathrm{NMR},{ }^{13} \mathrm{C} \mathrm{NMR}$ 和 MS 分析表明各产物的结构正 确.

本研究通过二取代烯基硼酸与 NCS 的反应建立了 一个制备取代的 $(Z)-3$-氯烯丙醇的新方法，该方法具有 操作简便, 反应条件温和, 产物构型保持且收率较高等 优点.

辅助材料(Supporting Information) (Z)-2-氯乙烯基-1己醇(1f) 的 ${ }^{1} \mathrm{H}-{ }^{1} \mathrm{H}$ NOESY 谱图. 这些材料可以免费从本 刊网站(http://sioc-journal.cn/)上下载.

\section{References}

[1] Steinhagen, H.; Corey, E. J. Angew. Chem., Int. Ed. 1999, 38, 1928.

[2] Williard, P. G.; Grab, L. A.; Laszlo, S. E. J. Org. Chem. 1983, 48, 1123.

[3] Marco, J. A.; Sanz-Cervera, J. F.; Manglano, E. Phytochemistry 1993, 33, 875.

[4] Kelth, C. W.; Lansing, Z., R. P. US 3300533, 1967 [Chem. Abstr. 1967, 66, 104810].

[5] Brown, H. C.; Subrahmanyam, C.; Hamaoka, T. J. Org. Chem. 1989, 54, 6068.

[6] Petasis, N. A.; Zavialov, I. A. Tetrahedron Lett. 1996, 37, 567.

[7] Fang, G.-H.; Yan, Z.-J.; Yang, J.; Deng, M.-Z. Synthesis 2006, 1148. 


\title{
辅助材料(Supporting Information)
}

\section{二取代烯基硼酸的氯化反应研究}

\author{
徐 衍 ${ }^{a}$ 李战雄 ${ }^{a}$ 李 慧 $b$ 杨 军*, \\ ( ${ }^{a}$ 苏州大学纺织与服装工程学院 苏州 215021) \\ ( ${ }^{b}$ 中国科学院上海有机化学研究所 上海 200032)
}

化合物(Z)-2-氯乙烯基-1-己醇(1f) ${ }^{1} \mathrm{H}-{ }^{1} \mathrm{H}$ NOESY 谱图如下:

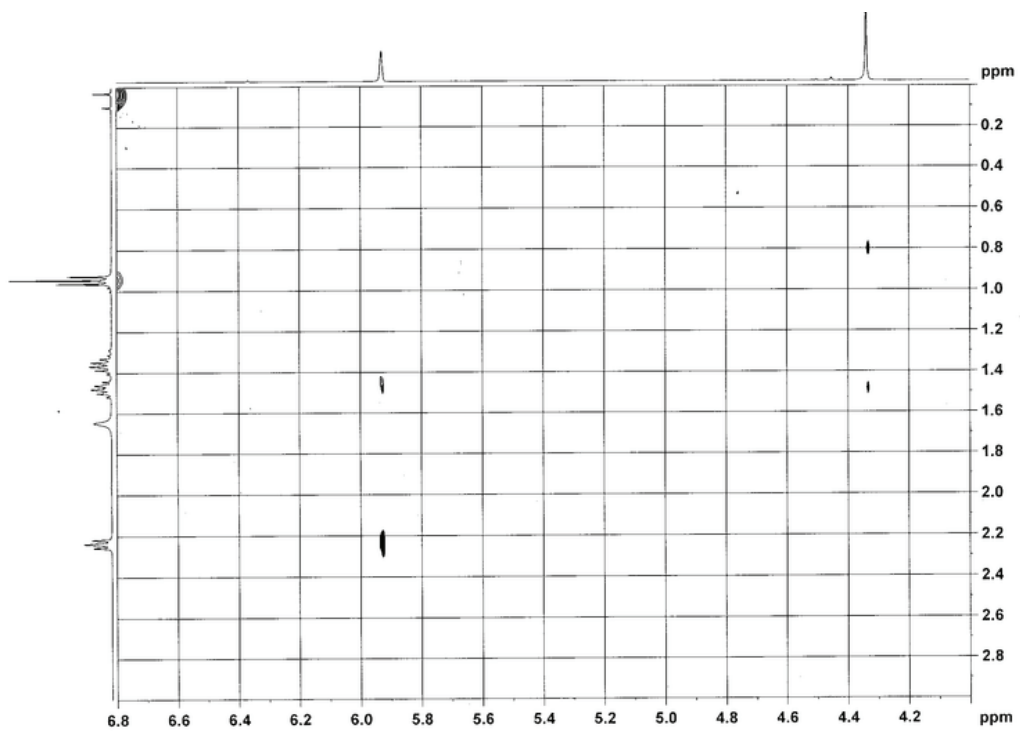

局部放大

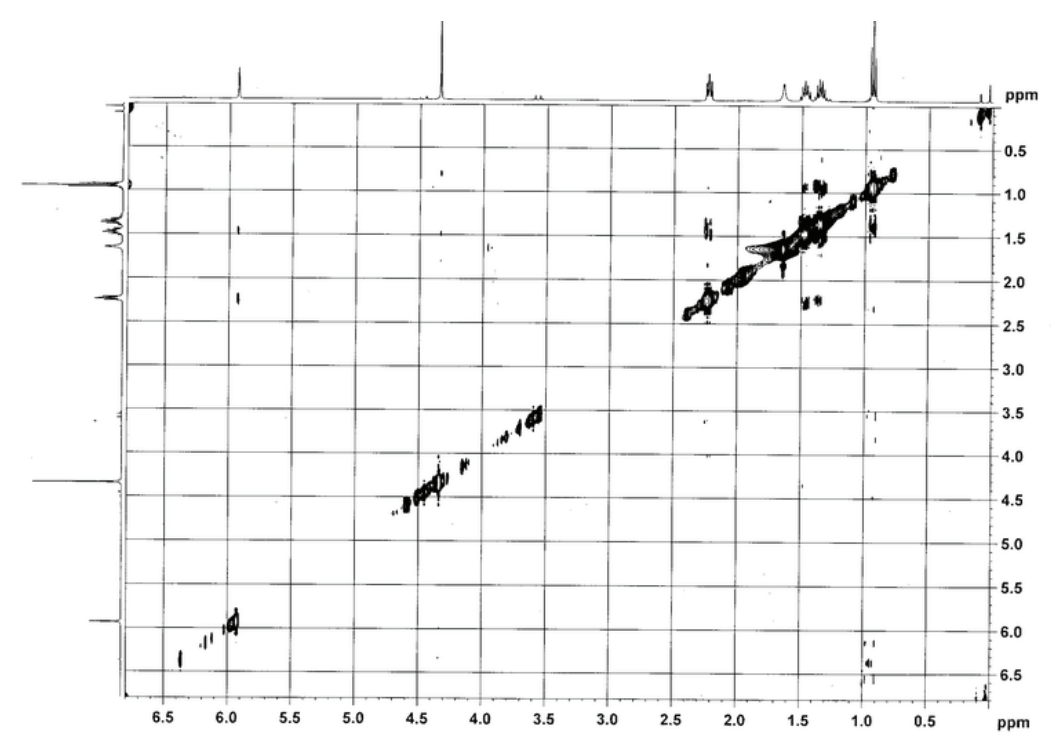

全谱

图 S1 化合物(Z)-2-氯乙烯基-1-已醇 (1f) ${ }^{1} \mathrm{H}-{ }^{1} \mathrm{H}$ NOESY 谱

Figure S1 $\quad{ }^{1} \mathrm{H}-{ }^{1} \mathrm{H}$ NOESY of (Z)-2-(chloromethylene)hexan-1-ol 